\title{
EMBEDDING NUCLEAR SPACES IN PRODUCTS OF AN ARBITRARY BANACH SPACE
}

\author{
STEPHEN A. SAXON
}

\begin{abstract}
It is proved that if $E$ is an arbitrary nuclear space and $F$ is an arbitrary infinite-dimensional Banach space, then there exists a fundamental (basic) system $\mathscr{V}$ of balanced, convex neighborhoods of zero for $E$ such that, for each $V$ in $\mathscr{V}$, the normed space $E_{V}$ is isomorphic to a subspace of $F$. The result for $F=l_{p}(1 \leqq p \leqq \infty)$ was proved by A. Grothendieck.
\end{abstract}

This paper is an outgrowth of an interest in varieties of topological vector spaces [2] stimulated by J. Diestel and S. Morris, and is in response to their most helpful discussions and questions. The main theorem, valid for arbitrary infinite-dimensional Banach spaces, was first proved by A. Grothendieck [3] (also, see [5, p. 101]) for the Banach spaces $l_{p}$ $(1 \leqq p \leqq \infty)$ and later by $\mathrm{J}$. Diestel for the Banach space $c_{0}$.

Our demonstration relies on two profound results of T. Kömura and Y. Kōmura [4] and C. Bessaga and A. Pełczyński [1], respectively:

(i) A locally convex space is nuclear if and only if it is isomorphic to a subspace of a product space $(s)^{I}$, where $I$ is an indexing set and $(s)$ is the Fréchet space of all rapidly decreasing sequences.

(ii) Every infinite-dimensional Banach space contains a closed infinitedimensional subspace which has a Schauder basis. ${ }^{1}$

Recall that for a balanced, convex neighborhood $V$ of zero in a locally convex space $E, E_{V}$ is a normed space which is norm-isomorphic to $\left(M,\left.p\right|_{M}\right)$, where $p$ is the gauge of $V$ and $M$ is a maximal linear subspace of $E$ on which $p$ is a norm; $\widetilde{E}_{V}$ is the completion of $E_{V}$. Denote by $(s)$ the nuclear Fréchet space of rapidly decreasing sequences, so that

$$
(s)=\left\{\left(\lambda_{n}\right): \sup _{n}\left|n^{k} \lambda_{n}\right|<\infty, k=1,2, \cdots\right\} \text {, }
$$

Received by the editors February 22, 1971 and, in revised form, October 25, 1971. AMS 1970 subject classifications. Primary 46A05, 46A35, 46B15.

Key words and phrases. Nuclear spaces, $(s), \tilde{E}_{V}$, product spaces, varieties, normisomorphic, Schauder basis.

1 This result was stated without proof in Banach's book (1932).

(c) American Mathematical Society 1972 
with the system of seminorms

$$
\left\{p_{k}\left(\left(\lambda_{n}\right)\right)=\sup _{n}\left|n^{k} \lambda_{n}\right|: k=1,2, \cdots\right\}
$$

LEMMA 1. Let $F$ be an arbitrary infinite-dimensional Banach space with a Schauder basis, and let $U$ be a neighborhood of zero in (s). There exists a balanced, convex neighborhood of zero $V \subset U$ such that $\widetilde{(s)}_{V}$ is normisomorphic to $F$.

PROOF. Let $\left\{x_{n}\right\}$ be a Schauder basis for $F$ with coefficient functionals $\left\{f_{n}\right\} \subset F^{\prime}$, and with $\left\|x_{n}\right\|=1(n=1,2, \cdots)$. By the uniform boundedness theorem, the (absolute) polar $\left\{f_{n}\right\}^{0}$ of $\left\{f_{n}\right\}$ is a neighborhood of zero, and its gauge $p$ is thus a continuous seminorm. Therefore $K\left\|\sum_{n=1}^{\infty} a_{n} x_{n}\right\| \geqq$ $p\left(\sum_{n=1}^{\infty} a_{n} x_{n}\right)=\sup _{n}\left|a_{n}\right|$ for each $\sum_{n=1}^{\infty} a_{n} x_{n} \in F$, where $K$ is some positive constant. For some $\varepsilon>0$ and positive integer $k$,

$$
V^{\prime}=\left\{x \in(s): p_{k}(x) \leqq \varepsilon\right\} \subset U .
$$

Define a norm $q$ on $(s)$ so that, for each $\left(\lambda_{n}\right) \in(s)$,

$$
\begin{aligned}
K\left(\sum_{n=1}^{\infty} n^{-2}\right) p_{k+2}\left(\left(\lambda_{n}\right)\right) & =K\left(\sum_{n=1}^{\infty} n^{-2}\right) \sup _{n}\left|n^{k+2} \lambda_{n}\right| \geqq K \sum_{n=1}^{\infty}\left|n^{k} \lambda_{n}\right| \\
& =K \sum_{n=1}^{\infty}\left\|n^{k} \lambda_{n} x_{n}\right\| \geqq K\left\|\sum_{n=1}^{\infty} n^{k} \lambda_{n} x_{n}\right\| \\
& \equiv q\left(\left(\lambda_{n}\right)\right) \geqq p\left(\sum_{n=1}^{\infty} n^{k} \lambda_{n} x_{n}\right)=p_{k}\left(\left(\lambda_{n}\right)\right) .
\end{aligned}
$$

Therefore $V=\{x \in(s): q(x) \leqq \varepsilon\} \subset V^{\prime} \subset U$ is a neighborhood of zero, and $(s)_{V}$ is norm-isomorphic to $((s), q)$, which is clearly norm-isomorphic to a dense subspace of $F$ by (*).

REMARK. It is clear from the proof that the map

$$
\left(\lambda_{n}\right) \rightarrow\left(\sum_{n=1}^{\infty} n^{k} \lambda_{n} x_{n}\right)_{k=1}^{\infty}
$$

embeds (s) in the product space $F \times F \times F \times \cdots$, and thus by (i) and (ii), each nuclear space can be embedded in some product of any given infinitedimensional Banach space.

LeMma 2. The space $(s)$ is isomorphic to $(s) \times(s)$.

Proof. The map $\left(\lambda_{n}\right) \rightarrow\left(\left(\lambda_{2 n-1}\right), \quad\left(\lambda_{2 n}\right)\right)$ is a linear, bicontinuous bijection from $(s)$ onto $(s) \times(s)$. 
THEOREM. Let $E$ be an arbitrary nuclear space and $F$ an arbitrary infinite-dimensional Banach space. Each neighborhood $U$ of zero in $E$ contains a balanced, convex neighborhood $V$ of zero such that $\tilde{E}_{V}$ is normisomorphic to a subspace of $F$.

Proof. Let $I$ be any indexing set, and $U$ a neighborhood of zero in the product space $E=(s)^{I}$. Thus there exists a finite subset $A$ of $I$ such that $U \supset W \times(s)^{B}$, where $B=I \sim A$ and $W$ is a neighborhood of zero in $(s)^{A}$. By (ii) there exists a closed infinite-dimensional subspace $F_{0}$ of $F$ which has a Schauder basis, and by Lemmas 1 and 2, there is a balanced, convex neighborhood of zero $V \subset W$ such that the completion of $\left((s)^{A}\right)_{V}$ is norm-isomorphic to $F_{0}$. Now $V^{\prime}=V \times(s)^{B}$ is a neighborhood of zero contained in $U$ such that $E_{V^{\prime}}$ is norm-isomorphic to $\left((s)^{A}\right)_{V}$ and the conclusion of the theorem holds for $E=(s)^{I}$. But then the conclusion clearly holds for any subspace of $(s)^{I}$, and hence for any nuclear space $E$, by (i).

COROllary. The variety ([2], [2a]) generated by any infinite-dimensional Banach space contains the variety of all nuclear spaces.

The author thanks Professor Pełczyński for the privilege and benefit of discussing this material with him.

\section{REFERENCES}

1. C. Bessaga and A. Pełczyński, On bases and unconditional convergence of series in Banach spaces, Studia Math. 17 (1958), 151-164. MR 22 \#5872.

2. J. Diestel, Sidney A. Morris and S. Saxon, Varieties of locally convex topological vector spaces, Bull. Amer. Math. Soc. 77 (1971), 799-803.

2a. - Varieties of linear topological spaces, Trans. Amer. Math. Soc. (to appear).

3. A. Grothendieck, Produits tensoriels topologiques et espaces nucléaires, Mem. Amer. Math. Soc. No. 16 (1955). MR 17, 763.

4. T. Kōmura and Y. Kōmura, Über die Einbettung der nuklaren Räume in $(s)^{\Lambda}$, Math. Ann. 162 (1965/66), 284-288. MR 32 \#6190.

5. H. H. Schaefer, Topological vector spaces, Macmillan, New York, 1966. MR 33 \#1738.

Department of Mathematics, University of Florida, Gainesville, Florida 32603 